\title{
BASIC SETS OF POLYNOMIALS FOR GENERALIZED BELTRAMI AND EULER-POISSON-DARBOUX EQUATIONS AND THEIR ITERATES ${ }^{1}$
}

\author{
E. P. MILES, JR. AND EUTIQUIO C. YOUNG
}

1. Introduction. This paper concerns basic sets of polynomial solutions for the class of partial differential equations in $m$ variables, $m \geqq 2$,

$$
L_{j}^{k}(u) \equiv\left(D_{m}+(-1) \sum_{i=1}^{j} D_{i}\right)^{k} u=0, \quad j=0,1 ; k=1,2, \cdots
$$

where

$$
D_{i}=\partial^{2} / \partial x_{i}^{2}+\left(\alpha_{i} / x_{i}\right)\left(\partial / \partial x_{i}\right) \quad \text { with } \quad \alpha_{i} \geqq 0 ; i=1, \cdots, m .
$$

The iterated operators $L_{j}^{k}$ are defined by the relations

$$
L_{j}^{s+1}(u)=L_{j}\left[L_{j}^{s}(u)\right], \quad s=1, \cdots, k-1 .
$$

When $\alpha_{1}=\cdots=\alpha_{m-1}=0$ and $\alpha_{m}>0, L_{j}(u)=0$ is known as the Beltrami or the Euler-Poisson-Darboux (EPD) equation according as $j=0$ or $j=1$. If $\alpha_{m}=0$ too, then $L_{0}(u)=0$ and $L_{1}(u)=0$ become the Laplace and wave equations, respectively. Basic sets of polynomial solutions for the Laplace and wave equations have been given in a number of papers [1]-[5]. In [6] Miles and Williams obtained basic sets of polynomials for the Beltrami and EPD equations from their result in [3]. In [7] the result of [3] was extended to form basic sets for the iterated Laplace and wave equations. Here we derive basic sets for (1) from the basic sets given in [7].

The Miles and Williams basic set of homogeneous polynomials of degree $n$ for the $k$-fold iterated Laplace equation $\Delta^{k} u=0$ $\left(\Delta=\sum_{i=1}^{m} \partial^{2} / \partial x_{i}^{2}\right)$ may be represented by

$$
\text { (2) } H_{a_{1}}^{n} \ldots a_{m}=\sum_{j=0}^{\left[\left(n-a_{m}\right) / 2\right]}(-1)^{j}\left(\begin{array}{c}
j+\left[a_{m} / 2\right] \\
{\left[a_{m} / 2\right]}
\end{array}\right) \Delta^{j}\left(x_{1}^{o_{1}} \cdots x_{m-1}^{a_{m-1}}\right) \frac{x_{m}^{2 j+a_{m}}}{\left(2 j+a_{m}\right) !} \text {, }
$$

where $a_{1}, \cdots, a_{m}$ are nonnegative integers such that $\sum_{i=1}^{m} a_{i}=n$ and $a_{m} \leqq 2 k-1$. In particular, when $a_{m}=0, H_{a_{1}}^{n}, \cdots, a_{m-1}, 0$ is harmonic,

Presented to the Society, January 24, 1967 under the title Basic sets of polynomials for iterated generalized Beltrami and EPD equations; received by the editors September 6, 1966.

1 This work was supported by NSF research grant GP-817. 
that is, it satisfies $\Delta u=0$. We shall prove that if for every index $i(1 \leqq i \leqq n)$ such that $\alpha_{i}>0$ we restrict the $a_{i}$ to be nonnegative even integers and replace $x_{i}^{2 s_{i}}$ by

$$
x_{i}^{\left(2 s_{i}\right)}=\frac{1 \cdot 3 \cdots\left(2 s_{i}-1\right)}{\left(1+\alpha_{i}\right) \cdots\left(2 s_{i}-1+\alpha_{i}\right)} x_{i}^{2 s_{i}},
$$

then (2) gives a basic set for $L_{0}^{k}(u)=0$ (or $L_{\mathbf{1}}^{\boldsymbol{k}}(u)=0$ if the factor $(-1)^{i}$ is deleted).

2. Basic set for $L_{0}^{s}(u)=0, s=1,2, \cdots, k$. We first observe that any polynomial solution of (1) must be even in the variable $x_{i}$ whenever $\alpha_{i}>0,1 \leqq i \leqq m$. Indeed, suppose $\alpha_{j}>0$ and suppose that $u(x)$ is a polynomial solution of (1) which contains odd powers of $x_{j}$, $x=\left(x_{1}, \cdots, x_{m}\right)$. Let $P\left(x^{\prime}\right)$, a polynomial of $x_{1}, \cdots, x_{j-1}, x_{j+1}$, $\cdots, x_{m}$, be the first nonvanishing coefficient of $x_{j}^{2 n+1}$ when $u(x)$ is arranged in ascending powers of $x_{j}$. Then the coefficient of $x_{j}^{2 n+1-2 k}$ in $L_{0}^{\boldsymbol{k}}(u)=0$ would be $(2 n+1) \cdots(2 n-2 k+3)\left(2 n+\alpha_{j}\right) \cdots$ $\left(2 n+\alpha_{j}-2 k+2\right) P\left(x^{\prime}\right)$, a nonvanishing function.

We assume that at least one of the $\alpha_{i}$ 's is not zero. In fact, by changing subscripts if necessary, we can assume $\alpha_{1}=\cdots=\alpha_{p}=0$, $\alpha_{p+1}>0, \cdots, \alpha_{m}>0,0 \leqq p \leqq m-1$. Let $a_{1}, \cdots, a_{p}, r_{p+1}, \cdots, r_{m}$ be a set of nonnegative in tegers satisfying the condition

$$
\sum_{i=1}^{p} a_{i}+\sum_{i=p+1}^{m} 2 r_{i}=N, \quad r_{m} \leqq k-1,
$$

and let

$$
\begin{aligned}
P_{a_{1}, \cdots, a_{p}, v_{p+1}, \cdots, r_{m}}^{N}= & \sum_{j=0}^{\left[\left(N-2 r_{m}\right) / 2\right]}(-1)^{j}\left(\begin{array}{c}
j+r_{m} \\
r_{m}
\end{array}\right) \\
& \cdot \Delta^{j}\left(x_{1}^{a_{1}} \cdots x_{p}^{a_{p}} x_{p+1}^{2 r_{p+1}} \cdots x_{m-1}^{2 r_{m-1}}\right) \frac{x_{m}^{2 j+2 r_{m}}}{\left(2 j+2 r_{m}\right) !} .
\end{aligned}
$$

Denote by $T_{i}(p+1 \leqq i \leqq m)$ the operator which replaces $x_{i}^{2 s_{i}}$ by $x_{i}^{\left(2 s_{i}\right)}$ (see (3)) in every term of the polynomial (5) and put $T=T_{p+1}$ $\cdots T_{m}{ }^{2}$ Then the operator $T$ applied to (5) replaces each factor $x_{p+1}^{2 s_{p+1}} \cdots x_{m}^{2 s_{m}}$ by $x_{p+1}^{\left(2 s_{p+1}\right)} \cdots x_{m}^{\left(2 s_{m}\right)}$. Moreover, from the fact that $D_{i} \cdot T_{i}=T_{i} \partial^{2} / \partial x_{i}^{2}$ and $D_{j} \cdot T_{k}=T_{k} \cdot D_{j}$ for $j \neq k$, we see that

${ }^{2}$ We are indebted to the referee for suggesting this notation and pointing out that the operator $T_{i}$ is a special case of a well-known operator treated by Lions, Operateurs de Delsarte et problèmes mixtes, Bull. Soc. Math. France 84 (1956); Proposition 2.1, p. 65 . 


$$
\begin{aligned}
L_{0} \cdot T & =\left(\sum_{i=1}^{p} \partial^{2} / \partial x_{i}^{2}+\sum_{i=p+1}^{m} D_{i}\right) T_{p+1} \cdots T_{m} \\
& =T \sum_{i=1}^{p} \partial^{2} / \partial x_{i}^{2}+\sum_{i=p+1}^{m} T_{p+1} \cdots D_{i} T_{i} \cdots T_{m} \\
& =T \sum_{i=1}^{p} \partial^{2} / \partial x_{i}^{2}+\sum_{i=p+1}^{m} T_{p+1} \cdots T_{i} \partial^{2} / \partial x_{i}^{2} \cdots T_{m} \\
& =T \sum_{i=1}^{p} \partial^{2} / \partial x_{i}^{2}+T \sum_{i=p+1}^{m} \partial^{2} / \partial x_{i}^{2} \\
& =T \Delta .
\end{aligned}
$$

Now let

$$
Q_{a_{1}, \cdots, a_{p}, r_{p+1}, \cdots, r_{m}}^{N}=T\left(P_{a_{1}, \cdots, a_{p}, r_{p+1}, \cdots, r_{m}}^{N}\right) .
$$

We assert that (7) forms a basic set for $L_{0}^{\mathbf{k}}(u)=0$.

LEMMA 1. $L_{0}\left(Q_{a_{1}}^{N}, \cdots, a_{p}, r_{p+1}, \cdots, r_{m-1}, 0\right)=0$.

This follows from (6) and the fact that $P_{a_{1}}^{N}, \cdots, a_{p}, r_{p+1}, \cdots, r_{m-1}, 0$ is harmonic.

LEMMA 2. $\quad L_{0}^{s}\left(Q_{a_{1}}^{N}, \cdots, a_{p}, r_{p+1}, \cdots, r_{m-1}, s\right)=Q_{a_{1}, \cdots, a_{p}, r_{p+1}, \cdots, r_{m-1}, 0}^{N-2 s}, \quad 0 \leqq 2 s$ $\leqq 2(k-1) \leqq N$.

Suppose that

$$
L_{0}^{j}\left(Q_{a_{1}}^{N}, \cdots, a_{p}, r_{p+1}, \cdots, r_{m-1}, j\right)=Q_{a_{1}, \cdots, a_{p}, r_{p+1}, \cdots, r_{m-1}, 0}^{N-2 j}
$$

$0 \leqq 2 j<2(k-1) \leqq N, a_{1}+\cdots+a_{p}+2 r_{p+1}+\cdots+2 r_{m-1}+2 j=N$. Then for $a_{1}+\cdots+a_{p}+2 r_{p+1}+\cdots+2 r_{m-1}+2(j+1)=N$, we have

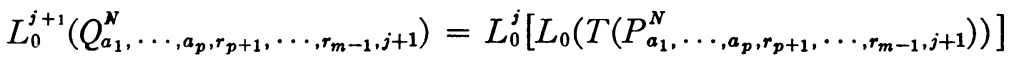

$$
\begin{aligned}
& =L_{0}^{j}\left[T \Delta P_{a_{1}}^{N}, \cdots, a_{p}, r_{p+1}, \cdots, r_{m-1}, j+1\right] \\
& =L_{0}^{j}\left[T\left(P_{a_{1}, \cdots, a_{p}, r_{p+1}}^{N-2}, \cdots, r_{m-1}, j\right)\right] \text {, } \\
& =L_{0}^{j}\left(Q_{a_{1}}^{N-2}, \cdots, a_{p}, r_{p+1}, \cdots, r_{m-1}, j\right) \\
& =Q_{a_{1}, \cdots, a_{p}, r_{p+1}, \cdots, r_{m-1}, 0}^{N-2-2 j}, \\
& \text { see (6) of }[7] \text {, }
\end{aligned}
$$

where $a_{1}+\cdots+a_{p}+2 r_{p+1}+\cdots+2 r_{m-1}+2 j=N-2$.

Now we verify that (7) forms a basic set for $L_{0}^{\boldsymbol{k}}(u)=0$. Consider first the case $2(k-1) \leqq N$. From Lemmas 1 and 2 it follows that all members of $(7)$ satisfy the equation $L_{0}^{\boldsymbol{k}}(u)=0$. So we need only to 
show that (7) has the correct number of independent polynomials. For a given integer $N \geqq 0$, it is clear that (7) has as many independent polynomials as there are distinct ways of choosing the set $a_{1}, \cdots, a_{p}$, $r_{p+1}, \cdots, r_{m}$ which satisfies (4). Let

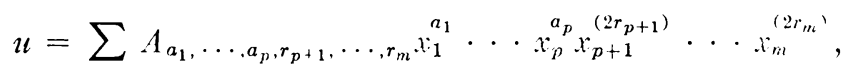

$a_{1}+\cdots+a_{p}+2 r_{p+1}+\cdots+2 r_{m}=N, 0 \leqq p \leqq m-1$, be any homogeneous polynomial of $x_{1}, \cdots, x_{m}$ of degree $N, N \geqq 2(k-1)$, which is even in the variables $x_{i}, p+1 \leqq i \leqq m$. Here we have already replaced each $x_{i}^{2 s_{i}}$ by $x_{i}^{\left(2 s_{i}\right)}$. Then every coefficient $A_{a_{1}, \cdots, a_{p}, \mathbf{r}_{p+1}, \cdots, \mathbf{r}_{m}}$ of $u$ can be represented, apart from constant factor, as

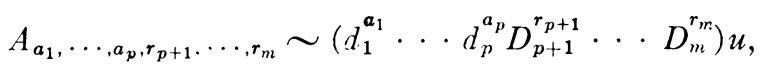

where $d_{i}=\partial / \partial x_{i}, i=1,2, \cdots, p$. If $L_{0}^{k}(u)=0$, so that

$$
D_{m}^{k} u=-\left(\sum \frac{k !}{a_{1} ! \cdots a_{p} ! r_{p+1} ! \cdots r_{m} !} d_{1}^{2 a_{1}} \cdots d_{p}^{2 a_{p}} D_{p+1}^{r_{p+1}} \cdots D_{m}^{r_{m}}\right) u
$$

where $a_{1}+\cdots+a_{p}+r_{p+1}+\cdots+r_{m}=k$ with $r_{m} \leqq k-1$, then every derivative of the form $\left(d_{1}^{a_{1}} \cdots d_{p}^{a_{p}} D_{p+1}^{r_{p+1}} \cdots D_{m}^{r_{m}}\right) u$ can be written in such a way that $D_{m}$ occurs no more than $(k-1)$ times. Thus, if $L_{0}^{k}(u)=0$, all coefficients of $u$ are linear combinations of the coefficients $A_{s_{1}, \cdots, s_{p}, t_{p+1}, \cdots, t_{m-1}, z}, 0 \leqq z \leqq k-1$, where $s_{1}+\cdots+s_{p}+2 t_{p+1}$ $+\cdots+2 z=N$ which coincides with (4). Therefore, for $2(k-1) \leqq N$, the set (7) is correctly numbered and hence forms a basic set for $L_{0}^{k}(u)=0$.

In the case $N<2(k-1)$, it is clear that all the members of (7) satisfy $L_{0}^{k}(u)=0$. In order to prove that $(7)$ has the correct number of polynomials, we examine (4) with $r_{m} \leqq[N / 2]$ under the following cases.

Case 1. $N=2 n, n \geqq 0$.

Suppose first that $p=2 q$; then for each $s, 0 \leqq s \leqq n$, where $s$ replaces $r_{m}$, only $2 v$ of the $a_{i}$ 's can be chosen as odd integers with $0 \leqq v \leqq[q, n]$. Here $[q, n]$ denotes the smaller of the integers $q$ and $n$. Hence, writing $a_{i}=2 r_{i}$ if $a_{i}$ is even and $a_{i}=2 r_{i}+1$ if $a_{i}$ is odd ( $\left.1 \leqq i \leqq p\right)$, we have $a_{1}+\cdots+a_{p}=2 r_{1}+\cdots+2 r_{p}+2 v$ so that (4) becomes $r_{1}+\cdots$ $+r_{m-1}=n-s-v$. Now for each $s, 0 \leqq s \leqq n$, the set (7) has

$$
\sum_{v=0}^{[q, n]}\left(\begin{array}{l}
2 q \\
2 v
\end{array}\right)\left(\begin{array}{c}
m+n-2-s-v \\
m-2
\end{array}\right)
$$

independent polynomials, since for each of the 


$$
\left(\begin{array}{c}
2 q \\
2 v
\end{array}\right)
$$

ways of choosing $2 v$ of the $a_{i}$ 's odd the set $P_{r_{1}}^{2 n} \ldots, r_{m-1}, s$ is seen to be generated by the monomials $x_{1}^{a_{1}} \cdots x_{p}^{a_{p}} x_{p+1}^{2 r_{p+1}} \cdots x_{m-1}^{2 r_{m-1}}$ with $a_{1}$ $+\cdots+a_{p}=2 r_{1}+\cdots+2 r_{p}+2 v$, where $x_{1}^{2 r_{1}} \cdots x_{m-1}^{2 r_{m-1}}$ are the individual terms appearing in $\left(\sum_{i=1}^{m-1} x_{i}^{2}\right)^{n-s-v}$. Therefore, for $N=2 n$ and $p=2 q$, the set ( 7$)$ consists of

$$
\sum_{s=0}^{n} \sum_{v=0}^{[q, n]}\left(\begin{array}{c}
2 q \\
2 v
\end{array}\right)\left(\begin{array}{c}
m+n-2-s-v \\
m-2
\end{array}\right)
$$

independent polynomials homogeneous of degree $2 n$. Here

$$
\left(\begin{array}{l}
a \\
b
\end{array}\right)
$$

is interpreted as zero whenever $a<b$.

If $p=2 q+1$, then ( 7$)$ will have

$$
\sum_{s=0}^{n} \sum_{v=0}^{[q, n]}\left(\begin{array}{c}
2 q+1 \\
2 v
\end{array}\right)\left(\begin{array}{c}
m+n-2-s-v \\
m-2
\end{array}\right)
$$

independent polynomials homogeneous of degree $2 n$.

Case 2. $N=2 n+1, n \geqq 0$.

In this case $p \neq 0$. This means that no polynomial of odd degree can satisfy $L_{0}^{\boldsymbol{k}}(u)=0$ when $\alpha_{i}>0, i=1,2, \cdots, m$. Again, suppose first that $p=2 q$; then $2 v+1$ of the $a_{i}$ 's must be chosen odd, $0 \leqq v$ $\leqq[q-1, n]$. Hence we can write $a_{1}+\cdots+a_{p}=2 r_{1}+\cdots+2 r_{p}$ $+2 v+1$ and again (4) reduces to $r_{1}+\cdots+r_{m-1}=n-s-v$. By the same argument as in Case 1 , we see that (7) has

$$
\sum_{s=0}^{n} \sum_{v=0}^{[q-1, n]}\left(\begin{array}{c}
2 q \\
2 v+1
\end{array}\right)\left(\begin{array}{c}
m+n-2-s-v \\
m-2
\end{array}\right)
$$

independent homogeneous polynomials of degree $2 n+1$.

For $p=2 q+1,(7)$ consists of

$$
\sum_{s=0}^{n} \sum_{v=0}^{[q, n]}\left(\begin{array}{c}
2 q+1 \\
2 v+1
\end{array}\right)\left(\begin{array}{c}
m+n-2-s-v \\
m-2
\end{array}\right)
$$

independent homogeneous polynomials.

Noting that for fixed $v$ the summands corresponding to $s>n-v$ are all zero, we can carry out the summation with respect to $s$ in the formulas (8)-(11). In fact, from (8) for example, we obtain 


$$
\begin{aligned}
\sum_{s=0}^{n} \sum_{v=0}^{[q, n]}\left(\begin{array}{c}
2 q \\
2 v
\end{array}\right)\left(\begin{array}{c}
m+n-2-s-v \\
m-2
\end{array}\right)=\sum_{v=0}^{[q, n]}\left(\begin{array}{l}
2 q \\
2 v
\end{array}\right) \\
\cdot \sum_{s=0}^{n-v}\left(\begin{array}{c}
m+n-2-s-v \\
m-2
\end{array}\right)=\sum_{v=0}^{[q, n]}\left(\begin{array}{c}
2 q \\
2 v
\end{array}\right)\left(\begin{array}{c}
m+n-v-1 \\
m-1
\end{array}\right),
\end{aligned}
$$

the number of independent polynomials in (7) when $N=2 n, n<k-1$, and $p=2 q$. Indeed, when $N<2(k-1)$ the basic set elements in case $N=2 n$ and $p=2 q$ could be chosen for each $v, 0 \leqq v \leqq[q, n]$, as the individual monomials $x_{1}^{a_{1}} \ldots x_{p}^{a_{p}} x_{p+1}^{2 r_{p+1}} \ldots x_{m}^{2 r_{m}}, a_{1}+\cdots+a_{p}=2 r_{1}$ $+\cdots+2 r_{p}+2 v$, where $x_{1}^{2 r_{1}} \cdots x_{m}^{2 r_{m}}$ are the individual terms appearing in $\left(\sum_{i=1}^{m} x_{i}^{2}\right)^{n-v}$ which has precisely

$$
\left(\begin{array}{c}
m+n-v-1 \\
m-1
\end{array}\right)
$$

elements. Members of basic set for the other cases can also be chosen in the same manner thus proving that (7) is correctly numbered.

Therefore, for given integers $N \geqq 0, k \geqq 1$, and the associated sets of nonnegative integers $a_{1}, \cdots, a_{p}, r_{p+1}, \cdots, r_{m}$ satisfying (4), $\left(2 r_{m} \leqq N\right.$ if $\left.N<2(k-1)\right)$, the set of polynomials given by (7) is a basic set for $L_{0}^{\mathbf{k}}(u)=0$.

3. Basic set for $L_{1}^{\boldsymbol{k}}(u)=0$. The corresponding basic set of polynomials for $L_{1}^{\mathbf{k}}(u)=0$ under the same assumption on the $\alpha_{i}$ 's as before may be deduced from (7) upon replacement of $x_{m}$ by $i x_{m}$. We have the same formula as (7) except for the absence of the factor $(-1)^{i}$. This fact can of course be established by the same procedure as in $\$ 2$ using (5) with the factor $(-1)^{i}$ deleted.

\section{REFERENCES}

1. M. H. Protter, Generalized spherical harmonics, Trans. Amer. Math. Soc. 63 (1948), 314-341.

2. - On a class of harmonic polynomials, Portugal. Math. 10 (1951), 11-22.

3. E. P. Miles, Jr. and Ernest Williams, A basic set of homogeneous harmonic polynomials in $k$ variables, Proc. Amer. Math. Soc. 6 (1955) 191-194.

4. J. Horváth, Singular integral operators and spherical harmonics, Trans. Amer. Math. Soc. 82 (1956), 52-63.

5. —_ Basic sets of polynomial solutions for partial differential equations, Proc. Amer. Math. Soc. 9 (1958), 569-575.

6. E. P. Miles, Jr. and Ernest Williams, $A$ basic set of polynomial solutions for the Euler-Poisson-Darboux and Beltrami equations, Amer. Math. Monthly 63 (1956), 401-404.

7. - Basic sets of polynomials for the iterated Laplace and wave equations, Duke Math. J. 26 (1959), 35-40.

Florida State University 